\title{
Time-Efficient Geometrically Non-Linear Finite Element Simulations of Thin Shell Deployable Structures
}

\author{
Narravula Harshavardhan Reddy* and Sergio Pellegrino ${ }^{\dagger}$ \\ California Institute of Technology, Pasadena, CA, 91125
}

\begin{abstract}
Isogeometric analysis of thin shells can provide higher continuity and exact geometric description. It is shown in the existing literature that isogeometric analysis converges with fewer degrees of freedom than $C^{0}$-continuous finite elements that use Langrange polynomial shape functions, but the speed of the solutions has not been previously assessed. In this research, the geometrically nonlinear bending of a thin shell deployable structure, a tape spring is studied, using both NURBS-based and $C^{0}$-continuous finite elements. The complex deformation of a tape spring makes it a perfect case study to compare the computational efficiency of the mentioned techniques. The simulations are carried out in the commercial software ABAQUS and LS-DYNA, and it is found that isogeometric analysis is at least three times slower than the $C^{0}$-continuous finite element methods.
\end{abstract}

\section{Nomenclature}

$\begin{array}{ll}p, q & =\text { polynomial degrees of NURBS basis functions } \\ \theta & =\text { total rotation applied } \\ M & =\text { reaction moment } \\ X, Y, Z & =\text { global Cartesian coordinates } \\ U, V, W & =\text { displacements in } X, Y, Z \text { directions } \\ \Theta_{X}, \Theta_{Y}, \Theta_{Z} & =\text { rotations about } X, Y, Z \text { axes } \\ \mathrm{RP} 1, \mathrm{RP} 2 & =\text { reference points } \\ L & =\text { length of tape spring } \\ R & =\text { radius of circular cross-section } \\ t & =\text { thickness of tape spring } \\ \alpha & =\text { angle subtended by the cross-section } \\ E & =\text { Young's modulus } \\ v & =\text { Poisson's ratio }\end{array}$

\section{Introduction}

$\mathrm{T}$ HIN shell structures have unique properties [1] and are widely used in many fields, including architecture and aerospace. There have been many studies [2] and for many years much emphasis has been placed on failure analysis, studying the initial buckling and post-buckling of thin shells. Recently, there has been an increasing number of studies and engineering applications in which the post-buckling behavior of thin shells [3, 4] is the key to achieving certain specific features. Examples include dampers [5, 6], actuators [7, 8], deployable structures [9]-11] and morphing structures [12, 13]. These applications require extensive, highly geometrically non-linear simulations and the computational effort for design optimization is currently mostly beyond reach. Enabling further developments in these areas requires new computational tools, and the present paper reports on a study of deployable thin shells using one of the latest developments in computational structural mechanics.

For background, it should be noted that the properties of thin shell structures are very sensitive to geometric imperfections [14], and finite element approximation of curved shell geometries introduces geometric imperfections because of inexact spatial discretization. Standard thin shell elements are formulated using Lagrange polynomials,

\footnotetext{
*Graduate Student, Graduate Aerospace Laboratories, MC 105-50. AIAA Student Member

${ }^{\dagger}$ Joyce and Kent Kresa Professor of Aerospace and Civil Engineering; Jet Propulsion Laboratory Senior Research Scientist; Co-Director, Space-Based Solar Power Project, Graduate Aerospace Laboratories, MC 105-50. AIAA Fellow. E-mail: sergiop@ caltech.edu
} 
which provide at most $C^{0}$ continuity at the element boundaries and make discretization errors inevitable. Very fine meshes are needed to reduce the effect of these errors, which is the source of higher computational costs.

Isogeometric analysis was recently developed [15] and is regarded as a promising solution to better approximate curved surfaces. This technique uses the same functions to approximate both the deformation of a shell and its initial geometry. Irrespective of the size of the mesh, the geometry of the shell is defined almost exactly, even for the most sparse choice of variables, thus greatly reducing the errors related to shape discretization. The shape functions-B-Splines, Non-Uniform Rational B-Splines (NURBS) or other parametrizations used in CAD—naturally span across multiple knot intervals (or 'elements') maintaining inter-element continuity which can be easily controlled. An early study of a shell element formulation based on isogeometric analysis is [16], followed by [17--22]. Despite the use of smooth, high-order shape functions, NURBS-based shell elements are not free from locking, although there have been successful attempts to avoid geometrical locking effects at the theory level [23, 24].

Several authors have already noted the advantages of isogeometric analysis to study buckling of plates and shells [25-28]. These studies have shown that NURBS-based analysis converges to accurate solutions and, in many cases, with fewer degrees of freedom compared to finite element analyses based on Lagrange polynomials. However, so far there has been no comparison of computational effort nor has there been any application of NURBS-based analysis to thin shell deployable structures. This article fills this gap by studying the quasi-static nonlinear bending of a tape spring - a straight and transversely curved strip of uniform thickness - that is the most basic deployable thin shell structure [29-31].

Tape springs can be deformed in their longitudinal planes to form localized elastic folds. If the bending is in the opposite sense to the natural transverse curvature, the tape spring undergoes a sudden snap-through buckling that results in the formation of a localized fold. When the bending is in the same sense of the transverse curvature, the fold formation is gradual. In both of these cases, the elastic fold is bounded on either sides by relatively undeformed sections of the tape spring. When released from the folded configuration, the tape spring locks back into its straight, undeformed position. Owing to the simplicity of their geometry, repeatable self-actuation, and ease of manufacturing, tape springs have found several applications in aerospace engineering [9, 29, 30, 32, 35]. The present study focuses on the simulation of opposite sense bending of an isotropic tape spring with initially circular cross-section, using both NURBS-based analysis software and standard finite element analysis. Convergence analyses are performed using both these techniques and the corresponding CPU times are compared. It will be shown that NURBS-based isogeometric analysis converges for fewer variables but is computationally more expensive.

The paper is organized as follows. Section [II discusses the commercial finite element software used for the study, along with the elements and solvers used. Section [II] discusses the properties of the tape spring chosen for the analysis and discusses the applied boundary conditions. Sections IV and V present the results obtained from the finite element simulations with $C^{0}$-continuous finite elements (with Lagrange polynomial shape functions) and NURBS-based elements, respectively. The conclusions of the research are presented in Section VI

\section{Software Tools}

Simulations of the displacement-controlled opposite-sense bending of an isotropic tape spring were carried out with the commercial finite element software ABAQUS and LS-DYNA. The analysis involves small strains and large rotations. The computational efficiency of the NURBS-based elements in LS-DYNA was compared with that of the

$C^{0}$-continuous shell elements in both ABAQUS and LS-DYNA. This section outlines the elements and solvers used for the simulations.

\section{A. ABAQUS}

S4R is a reduced integrated, 4-node, bilinear, flat shell element with six degrees of freedom per node and includes hourglass stabilization. It is a member of the ABAQUS library of general-purpose conventional shell elements and can be used for shells of any thickness. They are suitable for nonlinear analyses with large rotations and hence are ideal for the current study. For the behavior of S4R elements in popular benchmark problems for geometrically nonlinear analysis of shells, see [36].

Quasi-static simulations were performed in ABAQUS 2020 using the in-built Riks solver, with no parallel processing i.e., using only 1 CPU. All the default settings for the Riks solver, with automatic incrementation, were used. The initial arclength provided to the solver is 0.1 (10\% of the total arclength) unless specified otherwise. 


\section{B. LS-DYNA}

Quasi-static finite element simulations were also performed using the LS-DYNA (version R11.1) type-16 shell elements, recommended by the developers for implicit simulations. This element, also denoted as ELFORM=16, is a $2 \times 2$ integrated planar element based on Reissner-Mindlin kinematics and Hughes-Liu shell element formulation [37]. It has five degrees of freedom per node in the local coordinates.

NURBS-based isogeometric analysis is available in LS-DYNA through the keyword *Element_Shell_Nurbs_Patch. A surface may be both modeled and meshed with NURBS-based elements, and there is the option of using the LS-DYNA user interface or to import a NURBS patch in .iges format. The latter was done in this study using an open source toolbox [38]. The elements used were based on Hughes-Liu shell theory, without rotational degrees of freedom. Both full Gauss integration with $(p+1) \times(q+1)$ integration points and uniformly reduced Gauss integration with $p \times q$ integration points are investigated, with $p$ and $q$ denoting the polynomial degrees of the basis functions in the two parametric directions.

LS-DYNA offers three arc-length solvers: Crisfield, Ramm, and modified Crisfield, but they all failed to go past the limit point. Therefore, for all of the simulations in LS-DYNA, i.e. including both NURBS-based elements and bilinear planar elements, the Broyden-Fletcher-Goldfarb-Shanno (BFGS) quasi-Newton solver with automatic incrementation and no arc-length control was used. All solution control parameters and boundary conditions were kept constant throughout the analysis. LS-DYNA offers many control options for the solver and numerous control parameters must be chosen. The parameters that worked for all simulations presented in this study are listed in Table 1 . The default values were used for the parameters not mentioned in the table.

The keyword files were compiled on LS-Run with one CPU and double precision (note that LS-DYNA implicit does not support single precision).

\begin{tabular}{|cccccc|}
\hline \multicolumn{7}{|c}{ Control_Implicit_Auto } & & & \\
IAUTO & ITEOPT & ITEWIN & DTMIN & DTMAX & \\
1 & 25 & 20 & 0.01 & 1.0 & \\
\hline${ }^{*}$ Control_Implicit_General & & & \\
IMFLAG & DT0 & FORM & & & \\
1 & 0.05 & 0 & & & \\
\hline${ }^{*}$ Control_Implicit_Solution & & & \\
NSOLVR & ILIMIT & MAXREF & DCTOL & ECTOL & DNORM \\
12 & 21 & 25 & 0.0001 & 0.001 & 1 \\
\hline
\end{tabular}

Table 1 Parameters used for the keyword * Control in the LS-DYNA implicit simulations. Default values were used for any parameters not listed here.

\section{Case Study}

A tape spring is a longitudinally straight cylindrical shell with a circular arc cross-section. When the ends are rotated, the tape spring exerts monotonically increasing bending moments until a dynamic snap occurs and the moments suddenly decrease. From this point on, an elastic fold develops and the tape spring behaves like a constant-moment elastic hinge. This behavior is shown schematically in Fig. 11.c) and Fig. 6. The geometric properties (shown in Fig. 11) and the material properties (Young's modulus $E$ and Poisson's ratio $v$ ) of the specific tape spring that was studied are listed in Table 2

To facilitate the application of displacement boundary conditions, two reference points (massless nodes) RP1 and RP2 (in Fig. 1.a)) were introduced at the intersections of the centroidal axis of the tape spring, in the initial configuration, with the end cross-sectional planes. All the nodes (or control points) at an end cross-section are kinematically constrained to the reference point corresponding to that end. Therefore, the ends undergo rigid body rotations about the respective reference points.

In the ABAQUS simulations, RP2 was fixed in space while RP1 was allowed to translate along a straight line coinciding with the initial centroidal axis of the tape spring. A rotation of $\theta / 2$ around the positive $X$-axis was applied at both reference points, and the reaction moments at the ends were obtained from the analysis output. Under static equilibrium conditions, the two moments are equal in magnitude and opposite. 

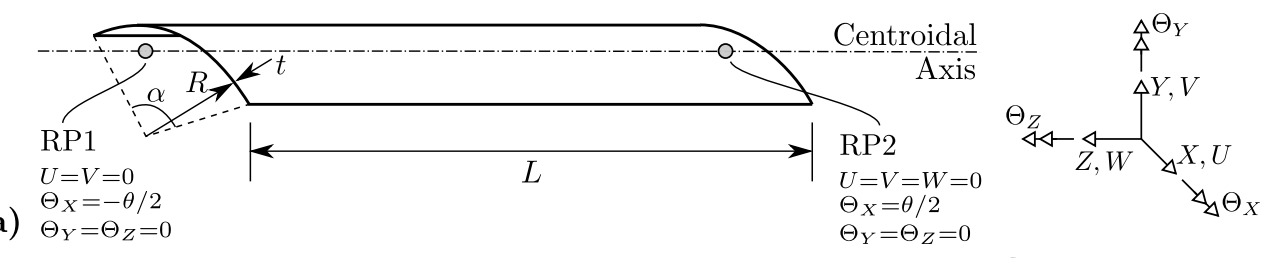

(a) $\begin{aligned} & \Theta_{X} \\ & \Theta_{Y}=\Theta_{Z}=0\end{aligned}$

(b) $\begin{aligned} & \Theta_{X} \\ & \Theta_{Y}=-\theta\end{aligned}=\Theta_{Z}=0$

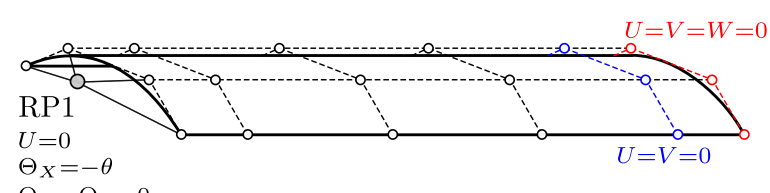

Fig. 1 (a) Schematic of tape spring of length $L$, radius of cross-section $R$, thickness $t$ and angle subtended $\alpha$. Reference points RP1 and RP2 are placed at the end cross-sections. (b) Boundary conditions on the nodes of rotation-free NURBS-based elements, (c) buckled tape spring under opposite sense bending, and (d) schematic depicting the total rotation applied.

In the case of rotation-free isogeometric analysis in LS-DYNA, the control points of one end cross-section were constrained to the reference point using the keywords *Extra_Nodes_Set or *Constrained_Nodal_Rigid_Body. Although the control points carry only displacement degrees of freedom, the reference point is allowed to carry rotations as well. Since it was not possible to obtain the reaction moments at a reference point constrained using the described keywords, the total rotation $\theta$ was applied at RP1 and the other end was clamped. The reaction moment $M$ (around the centroidal axis) at the clamped end was computed from the reaction forces in the $Z$ - direction.

A knot vector is called open or clamped when the first and the last knots are repeated $p+1$ times, $p$ being the degree of polynomial. When an open knot vector is used in a parametric direction, the NURBS surface is tangent to the control net (matrix of control points; faceted surface represented by dashed lines in Fig. 11b)) at either ends, in that direction [15]. This property was exploited in applying the fully clamped boundary condition shown in Fig. 11b).

\begin{tabular}{|c|c|c|c|c|c|}
\hline$L$ & $R$ & $t$ & $\alpha$ & $E$ & $v$ \\
\hline $200 \mathrm{~mm}$ & $10 \mathrm{~mm}$ & $0.1 \mathrm{~mm}$ & $110^{\circ}$ & $131 \mathrm{GPa}$ & 0.3 \\
\hline
\end{tabular}

Table 2 Properties of tape spring for the case study.

\section{Simulations with $C^{0}-$ Continuous Finite Elements}

The moment-rotation relationships for the opposite-sense bending of the tape spring, obtained from the simulations using uniform meshes of bilinear quadratic shell elements described in Section [I] are presented in this section.

Figure 2 shows the convergence analysis performed in ABAQUS using the S4R elements, and in LS-DYNA using the type-16 shell elements. As mentioned earlier, the resisting moment $M$ increases with the applied rotation $\theta$ until a limit point is reached. In this case, the peak moment of about $300 \mathrm{Nmm}$ occurs at a rotation $\theta \approx 12.5 \mathrm{deg}$. After going past the limit point, the tape spring undergoes a dynamic buckling and an elastic fold is formed. The resulting hinge offers a steady resistance of approx. $29 \mathrm{Nmm}$ against further rotation.

While arc-length solvers can trace the equilibrium path during the snap, the obtained data is non-physical and cannot be verified experimentally. Hence, this data was ignored when deciding on the convergence (Fig. 22a)). Quasi-Newton methods do not predict this behavior, so a sharp fall in the moment $M$ is seen in Fig. 2 (b).

Notice, in Fig. 22 that the results converge for a uniform mesh of $40 \times 15$ elements, i.e., 40 elements in the longitudinal direction and 15 in the circumferential direction, in the finite element simulations with both softwares. In addition, both softwares have converged to the same solution. To justify the choice of 15 elements along the arc, results have been presented for varying number of elements along the arc while keeping the number of elements along the length constant at 50, see Fig. 3 In the following, the $M-\theta$ curve for the $200 \times 40$ S4R mesh is used as a reference. 


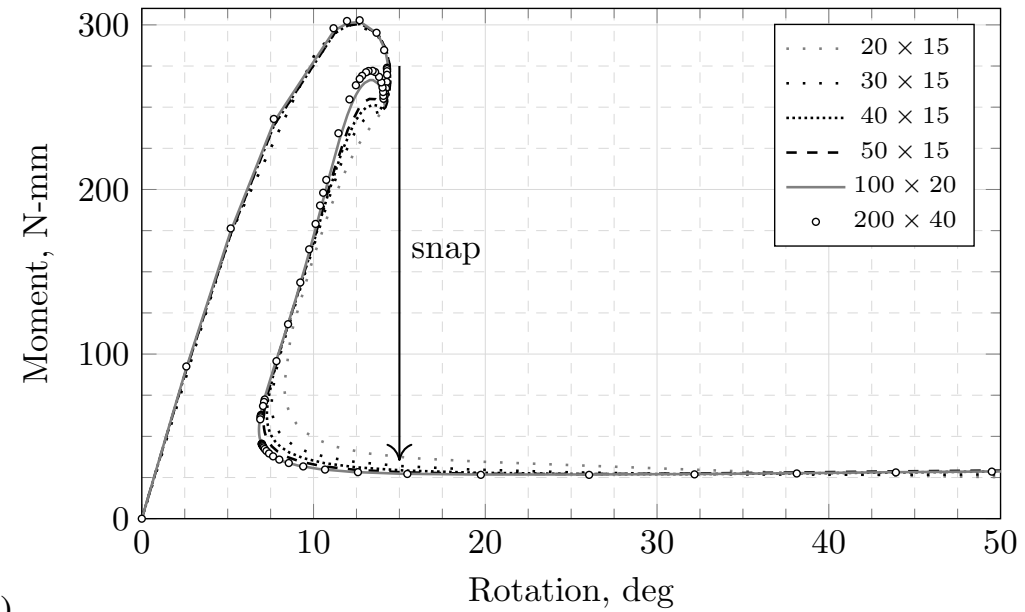

(a)

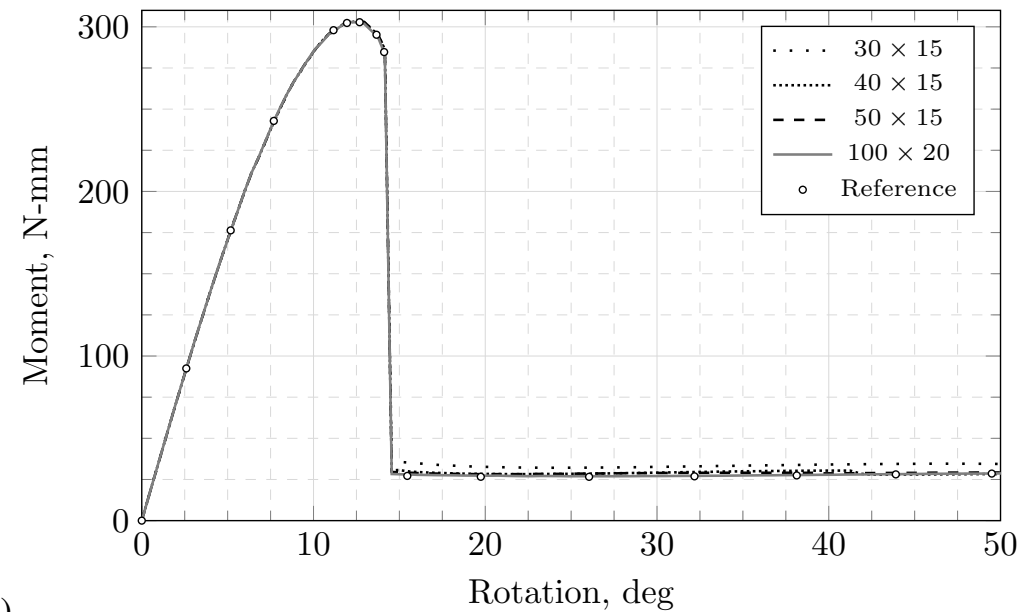

(b)

Fig. 2 Convergence analysis using uniform meshes of (a) ABAQUS S4R, and (b) LS-DYNA type-16 shell elements. The moment-rotation curve for $200 \times 40 \mathrm{~S} 4 \mathrm{R}$ mesh is chosen as the reference. In the legends, $m \times n$ refers to $\boldsymbol{m}$ and $\boldsymbol{n}$ uniformly distributed elements in the longitudinal and circumferential directions, respectively. 
The CPU times for the simulations presented in Fig. 2 are listed in Table 3 . The ABAQUS simulations for all of the mesh sizes listed in Table 3, except $30 \times 15$, failed for an initial arclength of 0.1 and hence this parameter was set to 0.05 for those mesh sizes. If the initial arc length of 0.1 had been successful, the computations would have been faster than the results listed in Table 3 Remember that S4R elements have 6 degrees of freedom per node while the type-16 shell elements of LS-DYNA have only 5. These results are compared against those for NURBS-based analyses presented in the following sections.

\begin{tabular}{|c|c|c|c|c|}
\hline \multirow{2}{*}{ Mesh size } & \multicolumn{2}{|c|}{ ABAQUS S4R } & \multicolumn{2}{c|}{ LS-DYNA shell type-16 } \\
\cline { 2 - 5 } & Degrees of freedom & CPU time (s) & Degrees of freedon & CPU time (s) \\
\hline $30 \times 15$ & 2976 & 24.7 & 2480 & 16 \\
$40 \times 15$ & 3936 & 28.9 & 3280 & 23 \\
$50 \times 15$ & 4896 & 45.2 & 4080 & 34 \\
$100 \times 20$ & 12726 & 121.9 & 10605 & 187 \\
\hline
\end{tabular}

Table 3 CPU times for ABAQUS and LS-DYNA simulations with uniform S4R and shell type-16 meshes.

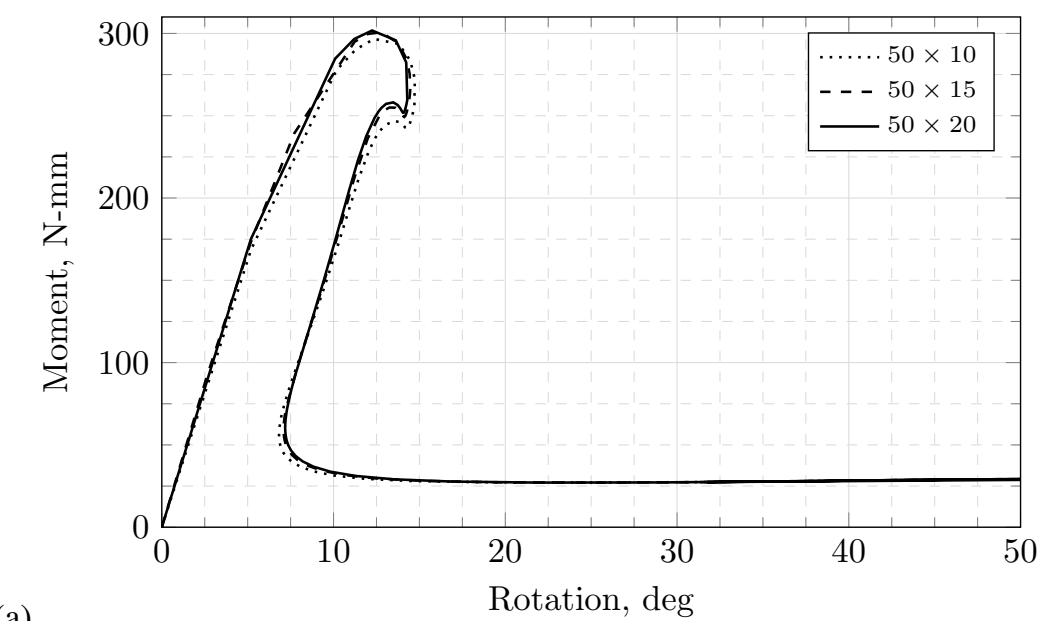

(a)

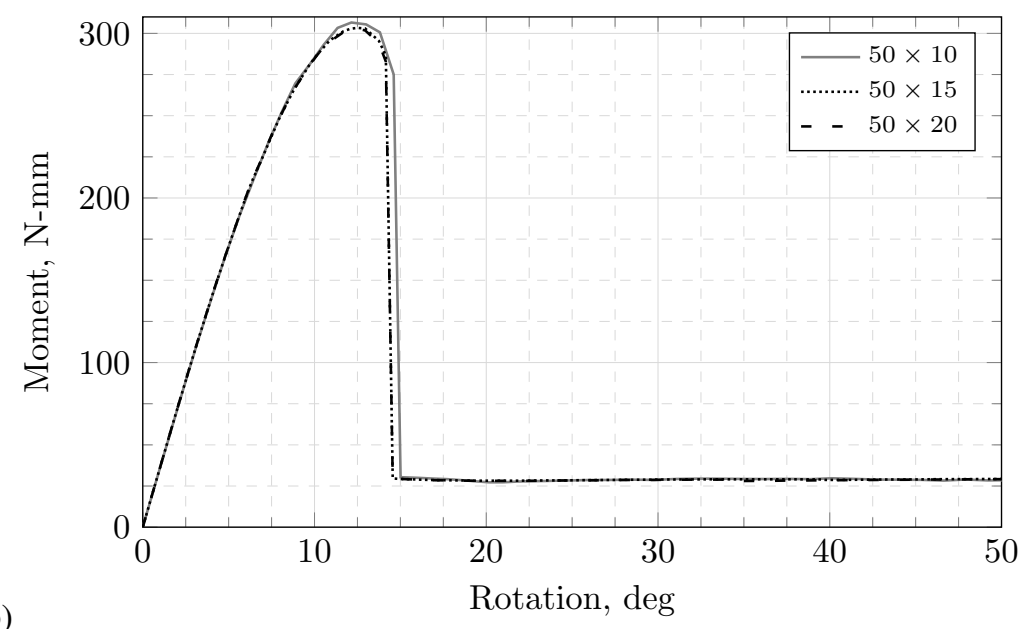

Fig. 3 Effect of the number of elements in the circumferential direction in (a) ABAQUS S4R and (b) LS-DYNA shell type-16 meshes. Along the length of the tape spring, the number of elements is kept constant at 50. 


\section{Simulations with NURBS-Based Elements}

This section presents the results obtained from the LS-DYNA implicit simulations using the NURBS-based elements. Open knot vectors were used with the multiplicity of all interior knots set to 1 . Therefore, the degree of continuity at the interior knots was $C^{p-1}$ and $C^{q-1}$ in the directions with polynomial degrees $p$ and $q$, respectively. If linear B-Splines had been used to construct the NURBS surface, continuity at the interior knots would have been $C^{0}$ and the surface would have interpolated all the control points. In addition, all the control points would have lied on the element boundaries. This leads to a faceted modeling of the curved surface and the exact geometric representation allowed by the splines would not be utilized.

The tape spring was meshed with either biquadratic ( $p=q=2)$ or bicubic ( $p=q=3$ ) elements, uniformly distributed over the surface. A fully integrated element has $(p+1) \times(q+1)$ integration points and uniformly reduced integration implies $p \times q$ integration points.

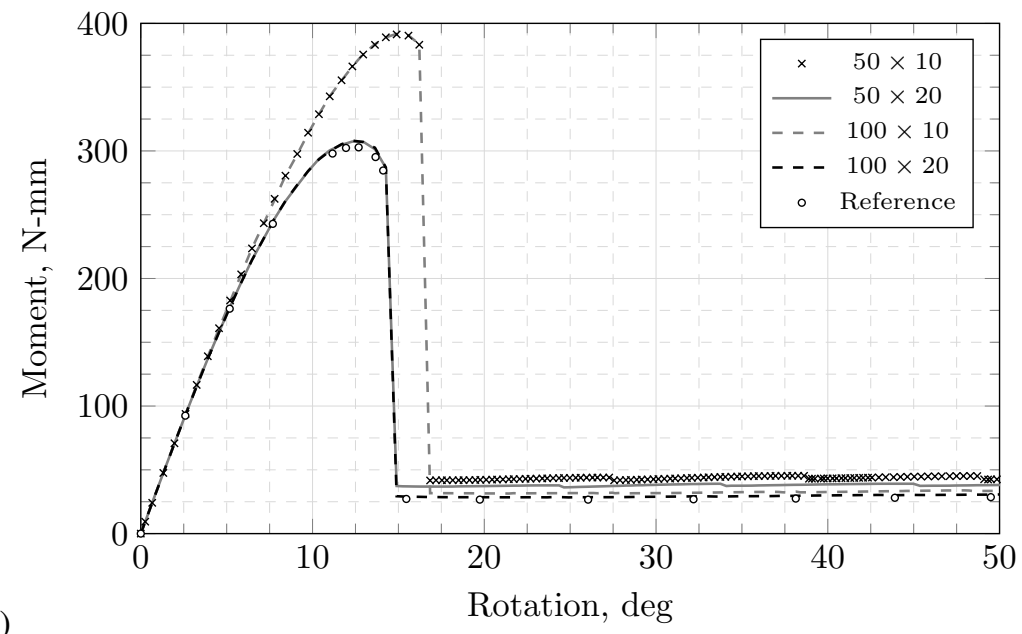

(a)

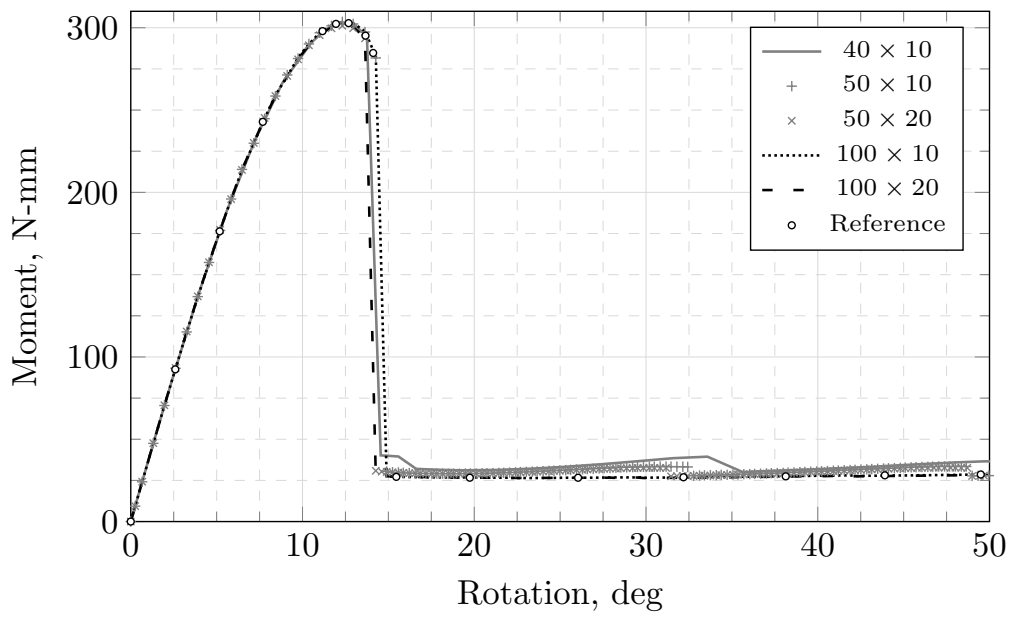

(b)

Fig. 4 Convergence analysis using (a) fully integrated and (b) reduced integrated biquadratic NURBS. In the legends, $m \times n$ refers to $m$ and $n$ uniformly distributed elements $((m+2)$ and $(n+2)$ control points) in the longitudinal and circumferential directions, respectively.

Figure 4 presents the moment-rotation curves obtained with biquadratic NURBS-based elements. Discretizing the arc with only 10 quadratic elements, independently of the number of elements along the length, leads to a stiffer response indicating membrane locking. This was eliminated by using a finer discretization in the circumferential direction or by reduced integration, Fig. 4(b), or by using higher order splines, Fig. 5 In Table 4 note that reduced integrated elements are computationally less expensive in most cases and, therefore, in the following only results obtained with reduced integrated elements are discussed. 
Figure 4(b) shows that 10 elements along the arc and 100 elements along the length are needed to obtain accurate results. Although having fewer elements along the length gives rise to an oscillating response in the post-buckling regime (for reasons unknown), the maximum deviation from the reference steady-state moment with respect to the peak moment is only $2 \%$. Therefore, convergence is assumed to have been achieved with the $50 \times 10$ mesh. The same oscillation is seen also in Fig. 5 for the $C^{2}$-continuous bicubic elements, except for the meshes with 100 elements along the length. The maximum deviation from the reference in this case is approx. $2.5 \%$ and it is assumed that convergence is achieved with a $50 \times 10$ mesh. The onset of buckling and the evolution of the elastic fold corresponding to the $50 \times 10$ biquadratic NURBS mesh are illustrated in Fig. 6.

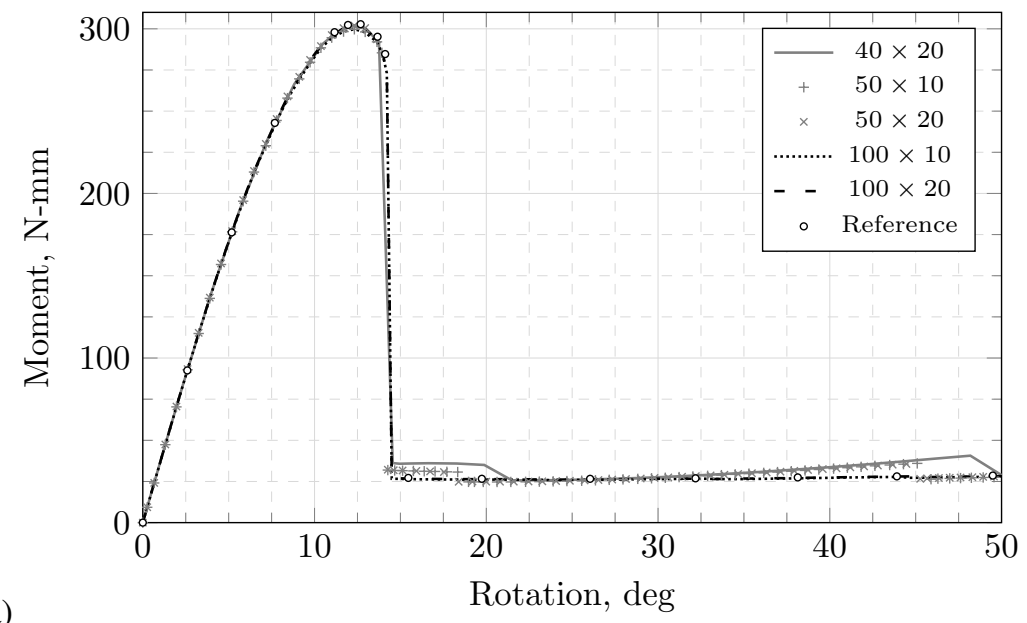

(a)

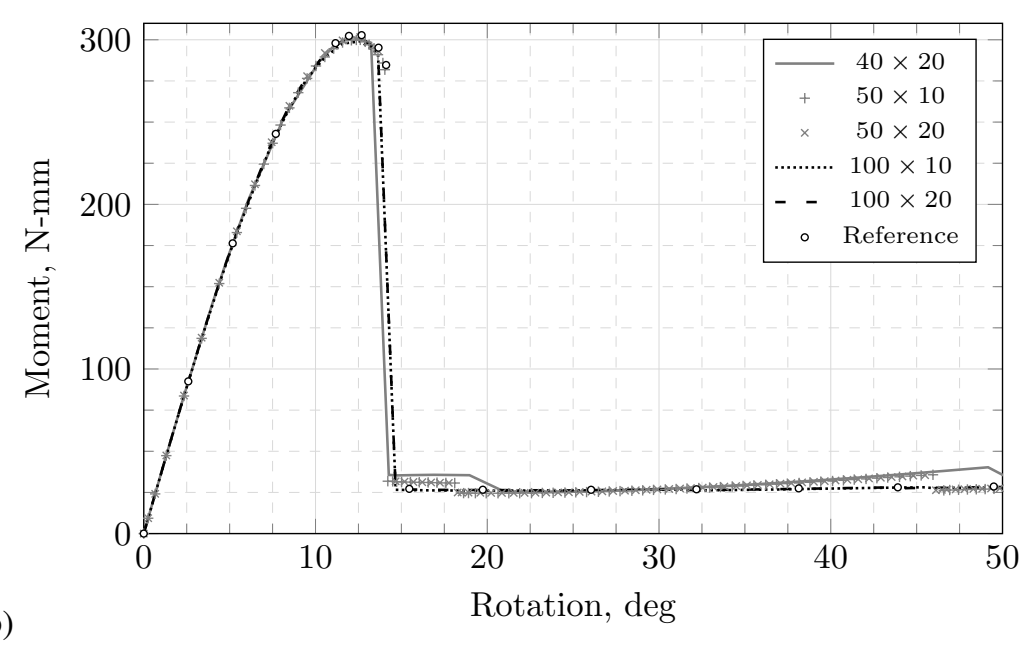

Fig. 5 Convergence analysis using (a) fully integrated and (b) reduced integrated bicubic NURBS. In the legends, $m \times n$ refers to $m$ and $n$ uniformly distributed elements $((m+3)$ and $(n+3)$ control points) in the longitudinal and circumferential directions, respectively.

The CPU times for the converging solutions were $76 \mathrm{~s}$ and $147 \mathrm{~s}$ for reduced integrated biquadratic and bicubic elements, respectively. Comparing these with the CPU times for $40 \times 15$ mesh of classical finite elements, see Table 3 , NURBS-based analysis was at least 4.75 times more expensive than the LS-DYNA type-16 shell elements and 3.08 times more expensive than the ABAQUS S4R elements. This proves that although NURBS-based elements converge for fewer degrees of freedom, they are computationally more expensive compared to classical finite elements. 


\begin{tabular}{|c|c|c|c|}
\hline Mesh size & Degrees of freedom & CPU time, fully integrated & CPU time, reduced integrated \\
\hline \multicolumn{3}{|c|}{ Biquadratic NURBS } \\
\hline $50 \times 10$ & 1873 & $81 \mathrm{~s}$ & $76 \mathrm{~s}$ \\
$50 \times 20$ & 3432 & $173 \mathrm{~s}$ & $143 \mathrm{~s}$ \\
$100 \times 10$ & 3672 & $250 \mathrm{~s}$ & $200 \mathrm{~s}$ \\
$100 \times 20$ & 6732 & $533 \mathrm{~s}$ & $409 \mathrm{~s}$ \\
\hline \multicolumn{3}{|c|}{ Bicubic NURBS } \\
\hline $50 \times 10$ & 2067 & $176 \mathrm{~s}$ & $147 \mathrm{~s}$ \\
$50 \times 20$ & 3657 & $379 \mathrm{~s}$ & $301 \mathrm{~s}$ \\
$100 \times 10$ & 4017 & $363 \mathrm{~s}$ & $459 \mathrm{~s}$ \\
$100 \times 20$ & 7107 & $1003 \mathrm{~s}$ & $838 \mathrm{~s}$ \\
\hline
\end{tabular}

Table 4 CPU times for LS-DYNA implicit simulations with NURBS-based elements.

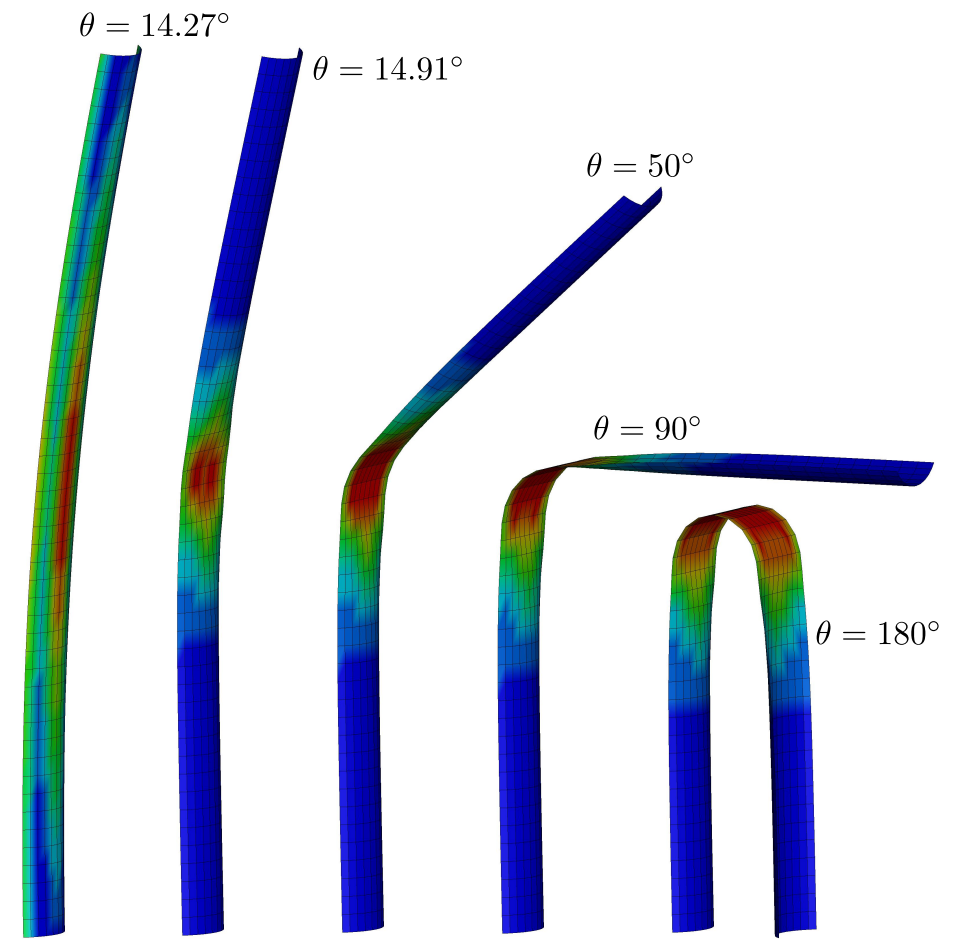

Fig. 6 Deformed configurations of tape spring meshed with $50 \times 10$ biquadratic NURBS-based elements. 


\section{Conclusions}

This research has applied isogeometric analysis to the geometrically nonlinear analysis of deployable thin shell structures. A tape spring under opposite sense bending was used as a means to compare the computational efficiency of higher order NURBS-based elements with that of the $C^{0}$-continuous finite elements. The elements compared were $C^{1}$-continuous biquadratic, and $C^{2}$-continuous bicubic NURBS-based elements in LS-DYNA; $C^{0}$-continuous 4-node bilinear elements in ABAQUS; and fully integrated bilinear quadrilateral (type-16) elements of LS-DYNA. It was observed that although the higher-order and smooth NURBS-based elements converge with fewer degrees of freedom, they are computationally more expensive than the $C^{0}$-continuous finite elements utilizing Lagrange polynomial shape functions.

\section{Acknowledgments}

This research was funded by the Space Solar Power Project at Caltech.

\section{References}

[1] Miura, K., and Pellegrino, S., Forms and Concepts for Lightweight Structures, Cambridge University Press, 2020.

[2] Bushnell, D., and Bushnell, W. D., shellbuckling.com, 2015.

[3] Hu, N., and Burgueño, R., "Buckling-induced smart applications: recent advances and trends," Smart Materials and Structures, Vol. 24, No. 6, 2015, p. 063001.

[4] Reis, P. M., "A perspective on the revival of structural (in) stability with novel opportunities for function: from buckliphobia to buckliphilia," Journal of Applied Mechanics, Vol. 82, No. 11, 2015.

[5] Bisagni, C., and Cordisco, P., "An experimental investigation into the buckling and post-buckling of CFRP shells under combined axial and torsion loading," Composite Structures, Vol. 60, No. 4, 2003, pp. 391 - 402. https://doi.org/https: //doi.org/10.1016/S0263-8223(03)00024-2. URL http://www.sciencedirect.com/science/article/pii/S0263822303000242.

[6] Galletly, D. A., and Guest, S. D., "Bistable composite slit tubes. II. A shell model," International Journal of Solids and Structures, Vol. 41, No. 16, 2004, pp. 4503 - 4516. https://doi.org/https://doi.org/10.1016/j.ijsolstr.2004.02.037, URL http://www.sciencedirect.com/science/article/pii/S0020768304000939

[7] Lazarus, A., and Reis, P. M., "Soft actuation of structured cylinders through auxetic behavior," Advanced Engineering Materials, Vol. 17, No. 6, 2015, pp. 815-820.

[8] Loukaides, E., Smoukov, S., and Seffen, K., "Magnetic actuation and transition shapes of a bistable spherical cap," International Journal of Smart and Nano Materials, Vol. 5, No. 4, 2014, pp. 270-282. https://doi.org/10.1080/19475411.2014.997322. URL https://doi.org/10.1080/19475411.2014.997322

[9] Pellegrino, S., Deployable structures, Vol. 412, Springer, 2014.

[10] Leclerc, C., Wilson, L. L., Bessa, M. A., and Pellegrino, S., "Characterization of ultra-thin composite triangular rollable and collapsible booms," 4th AIAA Spacecraft Structures Conference, 2017, p. 0172.

[11] Sakovsky, M., and Pellegrino, S., "Closed cross-section dual-matrix composite hinge for deployable structures," Composite Structures, Vol. 208, 2019, pp. 784-795.

[12] Dai, F., Li, H., and Du, S., "A multi-stable lattice structure and its snap-through behavior among multiple states," Composite Structures, Vol. 97, 2013, pp. 56 - 63. https://doi.org/https://doi.org/10.1016/j.compstruct.2012.10.016 URL http://www. sciencedirect.com/science/article/pii/S0263822312004953.

[13] Pirrera, A., Avitabile, D., and Weaver, P., "On the thermally induced bistability of composite cylindrical shells for morphing structures," International Journal of Solids and Structures, Vol. 49, No. 5, 2012, pp. 685 - 700. https://doi.org/https: //doi.org/10.1016/j.ijsolstr.2011.11.011, URL http://www.sciencedirect.com/science/article/pii/S0020768311003933

[14] Calladine, C., "Understanding imperfection-sensitivity in the buckling of thin-walled shells," Thin-Walled Structures, Vol. 23, No. 1, 1995, pp. 215 - 235. https://doi.org/https://doi.org/10.1016/0263-8231(95)00013-4. URL http://www.sciencedirect.com/ science/article/pii/0263823195000134, buckling Strength of Imperfection-sensitive Shells. 
[15] Hughes, T. J., Cottrell, J. A., and Bazilevs, Y., "Isogeometric analysis: CAD, finite elements, NURBS, exact geometry and mesh refinement," Computer methods in applied mechanics and engineering, Vol. 194, No. 39-41, 2005, pp. 4135-4195.

[16] Kiendl, J., Bletzinger, K.-U., Linhard, J., and Wüchner, R., "Isogeometric shell analysis with Kirchhoff-Love elements," Computer Methods in Applied Mechanics and Engineering, Vol. 198, No. 49, 2009, pp. 3902 - 3914. https://doi.org/https: //doi.org/10.1016/j.cma.2009.08.013, URL http://www.sciencedirect.com/science/article/pii/S0045782509002680

[17] Benson, D., Bazilevs, Y., Hsu, M.-C., and Hughes, T., “A large deformation, rotation-free, isogeometric shell," Computer Methods in Applied Mechanics and Engineering, Vol. 200, No. 13-16, 2011, pp. 1367-1378.

[18] Echter, R., Oesterle, B., and Bischoff, M., "A hierarchic family of isogeometric shell finite elements," Computer Methods in Applied Mechanics and Engineering, Vol. 254, 2013, pp. 170-180.

[19] Oesterle, B., Ramm, E., and Bischoff, M., "A shear deformable, rotation-free isogeometric shell formulation," Computer Methods in Applied Mechanics and Engineering, Vol. 307, 2016, pp. 235-255.

[20] Oesterle, B., Sachse, R., Ramm, E., and Bischoff, M., "Hierarchic isogeometric large rotation shell elements including linearized transverse shear parametrization," Computer Methods in Applied Mechanics and Engineering, Vol. 321, 2017, pp. 383-405.

[21] Nguyen-Thanh, N., Zhou, K., Zhuang, X., Areias, P., Nguyen-Xuan, H., Bazilevs, Y., and Rabczuk, T., "Isogeometric analysis of large-deformation thin shells using RHT-splines for multiple-patch coupling," Computer Methods in Applied Mechanics and Engineering, Vol. 316, 2017, pp. 1157-1178.

[22] Leonetti, L., Liguori, F., Magisano, D., and Garcea, G., "An efficient isogeometric solid-shell formulation for geometrically nonlinear analysis of elastic shells," Computer Methods in Applied Mechanics and Engineering, Vol. 331, 2018, pp. 159-183.

[23] Oesterle, B., Bieber, S., Sachse, R., Ramm, E., and Bischoff, M., "Intrinsically locking-free formulations for isogeometric beam, plate and shell analysis," PAMM, Vol. 18, No. 1, 2018, p. e201800399.

[24] Bieber, S., Oesterle, B., Ramm, E., and Bischoff, M., "A variational method to avoid locking-independent of the discretization scheme," International Journal for Numerical Methods in Engineering, Vol. 114, No. 8, 2018, pp. 801-827.

[25] Guo, Y., Do, H., and Ruess, M., "Isogeometric stability analysis of thin shells: From simple geometries to engineering models," International Journal for Numerical Methods in Engineering, Vol. 118, No. 8, 2019, pp. 433-458.

[26] Praciano, J. S. C., Barros, P. S. B., Barroso, E. S., Parente, E., Áurea Silva de Holanda, and Sousa, J. B. M., "An isogeometric formulation for stability analysis of laminated plates and shallow shells," Thin-Walled Structures, Vol. 143, 2019, p. 106224. https://doi.org/https://doi.org/10.1016/j.tws.2019.106224, URL http://www.sciencedirect.com/science/article/pii// S0263823118306839

[27] Le-Manh, T., and Lee, J., "Postbuckling of laminated composite plates using NURBS-based isogeometric analysis," Composite Structures, Vol. 109, 2014, pp. 286 - 293. https://doi.org/https://doi.org/10.1016/j.compstruct.2013.11.011, URL http: //www.sciencedirect.com/science/article/pii/S0263822313005916.

[28] Shojaee, S., Valizadeh, N., Izadpanah, E., Bui, T., and Vu, T.-V., "Free vibration and buckling analysis of laminated composite plates using the NURBS-based isogeometric finite element method," Composite Structures, Vol. 94, No. 5, 2012 , pp. 1677 1693. https://doi.org/https://doi.org/10.1016/j.compstruct.2012.01.012, URL http://www.sciencedirect.com/science/article/pii/ S026382231200027X

[29] Pellegrino, S., "Folding and deployment of thin shell structures," Extremely deformable structures, Vol. 562, edited by D. Bigoni, Springer, Vienna, 2015, pp. 179-267.

[30] Seffen, K., and Pellegrino, S., "Deployment dynamics of tape springs," Proceedings of the Royal Society of London. Series A: Mathematical, Physical and Engineering Sciences, Vol. 455, No. 1983, 1999, pp. 1003-1048.

[31] Mallikarachchi, H., and Pellegrino, S., "Design of ultrathin composite self-deployable booms," Journal of Spacecraft and Rockets, Vol. 51, No. 6, 2014, pp. 1811-1821.

[32] Seffen, K., You, Z., and Pellegrino, S., "Folding and deployment of curved tape springs," International Journal of Mechanical Sciences, Vol. 42, No. 10, 2000, pp. 2055 - 2073. https://doi.org/https://doi.org/10.1016/S0020-7403(99)00056-9. URL http://www.sciencedirect.com/science/article/pii/S0020740399000569

[33] Watt, A. M., “Deployable structures with self-locking hinges,” Ph.D. thesis, University of Cambridge, 2003. 
[34] Seffen, K., Wang, B., and Guest, S., "Folded orthotropic tape-springs," Journal of the Mechanics and Physics of Solids, Vol. 123, 2019, pp. 138 - 148. https://doi.org/https://doi.org/10.1016/j.jmps.2018.09.017. URL http://www.sciencedirect.com/ science/article/pii/S0022509618305246 the N.A. Fleck 60th Anniversary Volume.

[35] Wilson, L., Gdoutos, E. E., and Pellegrino, S., "Tension-Stabilized Coiling of Isotropic Tape Springs," International Journal of Solids and Structures, Vol. 188-189, 2020, pp. 103 - 117. https://doi.org/https://doi.org/10.1016/j.ijsolstr.2019.09.010, URL http://www.sciencedirect.com/science/article/pii/S0020768319304226.

[36] Sze, K., Liu, X., and Lo, S., "Popular benchmark problems for geometric nonlinear analysis of shells," Finite Elements in Analysis and Design, Vol. 40, No. 11, 2004, pp. 1551 - 1569. https://doi.org/https://doi.org/10.1016/j.finel.2003.11.001, URL http://www.sciencedirect.com/science/article/pii/S0168874X0300218X

[37] Hughes, T. J., and Liu, W. K., "Nonlinear finite element analysis of shells: Part I. Three-dimensional shells," Computer methods in applied mechanics and engineering, Vol. 26, No. 3, 1981, pp. 331-362.

[38] Spink, M., "NURBS toolbox for MATLAB," https://octave.sourceforge.io/nurbs/overview.html. 